\title{
Microwave Subsurface Imaging Technology for Damage Detection
}

\author{
Yoo Jin Kim, A.M.ASCE${ }^{1}$; Luis Jofre ${ }^{2}$; Franco De Flaviis ${ }^{3}$; and Maria Q. Feng, M.ASCE ${ }^{4}$
}

\begin{abstract}
This paper presents a technology for detecting invisible damage inside concrete, which is based on reconstruction of dielectric profile (image) of the concrete illuminated with microwaves sent from and received by antenna arrays controlled by specialized software. The imaging system developed in this study consists of an $8 \times 8$ transmitting and an $8 \times 8$ receiving arrays, an innovative numerical bifocusing operator for improving image resolution, and imaging software for reconstructing a two-dimensional image from the scattered field. The effectiveness of the developed technology in detecting steel and voids inside concrete has been demonstrated through numerical simulation and experiments.
\end{abstract}

DOI: 10.1061/(ASCE)0733-9399(2004)130:7(858)

CE Database subject headings: Microwaves; Imaging techniques; Antennas; Concrete structures; Damage assessment; Nondestructive tests.

\section{Background and the Proposed Technology}

Damage assessment of concrete structures relies heavily on visual inspections. However, it is difficult to assess the extent of damage developed inside the concrete based on concrete surface cracks. Internal and invisible damage (such as voids, cracks, delaminations, and debonds) caused by corrosions, earthquakes, and others is of significant safety concern.

Use of microwave for nondestructive evaluation of concrete structures has been recently explored (e.g., Bois et al. 1998; Huston et al. 2000; Rhim and Büyüköztürk 2000; Kharkovsky and Atis 2003). The writers developed surface-focused microwave imaging technology in previous work and demonstrated its effectiveness in detecting debonds in fiber-reinforced-polymerjacketed concrete structures (Feng et al. 2002; Kim 2002). The image is constructed through scanning, point-by-point, of the structural surface using one pair of dielectric lenses, whose fixed focusing distance makes it difficult to detect damage at an arbitrary depth.

The objective of this study is to detect and quantify the invisible damage/objects inside concrete through subsurface imaging. In order to accomplish the objective, a subsurface-focused microwave imaging technology using transmitting and receiving an-

\footnotetext{
${ }^{1}$ Post Graduate Researcher, Dept. of Civil and Environmental Engineering, Univ. of California, Irvine, CA 92697-2175. E-mail: ykim@newportsensors.com

${ }^{2}$ Professor, Dept. of Signal Theory and Communications, Technical Univ. of Catalonia, Barcelona, Spain.

${ }^{3}$ Assistant Professor, Dept. of Electrical and Computer Engineering, Univ. of California, Irvine, CA 92697-2175.

${ }^{4}$ Professor, Dept. of Civil and Environmental Engineering, Univ. of California, Irvine, CA 92697-2175.

Note. Associate Editor: Eric N. Landis. Discussion open until December 1, 2004. Separate discussions must be submitted for individual papers. To extend the closing date by one month, a written request must be filed with the ASCE Managing Editor. The manuscript for this paper was submitted for review and possible publication on May 14, 2002; approved on January 14, 2004. This paper is part of the Journal of Engineering Mechanics, Vol. 130, No. 7, July 1, 2004. CASCE, ISSN 0733-9399/ 2004/7-858-866/\$18.00.
}

tenna arrays was developed, in which the waves are focused by software, rather than lenses. This enables much more flexibility for detecting damage deep below the structural surface and higher efficiency for speedy imaging. Fig. 1 depicts the system configuration of the proposed subsurface imaging technology. Cylindrical antenna arrays are used for transmitting and receiving microwave signals, which are focused to a measurement point inside concrete by software, a numerical bifocusing operator focusing both receiving and transmitting waves. This makes it possible to quickly sweep the focusing point, scan a large area involving many measurement points, and form a tomographic image. This paper first describes the two-dimensional (2D) tomographic image reconstruction algorithm involving the numerical bifocusing procedure, then presents the design and fabrication of prototype antenna arrays, and finally demonstrates the effectiveness of the proposed subsurface imaging technology in detecting voids and steels inside concrete through numerical and experimental studies.

\section{Image Reconstruction Algorithm}

\section{Analytical Formulation}

As illustrated in Fig. 1, the antenna array consists of $N_{n} \times N_{m}$ antenna elements, $N_{n}$ forming a transmitting and $N_{m}$ forming a receiving array. A $N_{n} \times N_{m}$ measurement matrix can be obtained as follows: for every selected transmitting element, the receiving array is scanned obtaining an $N_{m}$-measurement column, then the procedure is repeated for the rest of the $N_{n}$ transmitting elements. Due to the basic 2D characteristic of the geometry under study, each element consists of a long vertical antenna array with a uniform current distribution. In practical terms, the length of the vertical antenna array $H$ has to be greater than the transversal dimension of the focusing area $L$. Following the electromagnetic compensation principle, the illumination of an inclusion (such as an air void) induces an equivalent electric current distribution, $J_{e q}\left(x_{0}, y_{0}, z_{0}\right)$, and this distribution creates an electromagnetic image of the object in image reconstruction (Gironés et al. 1987). The reconstruction algorithm forms an image point by means of the synthesis of two focused arrays (transmitting and receiving 


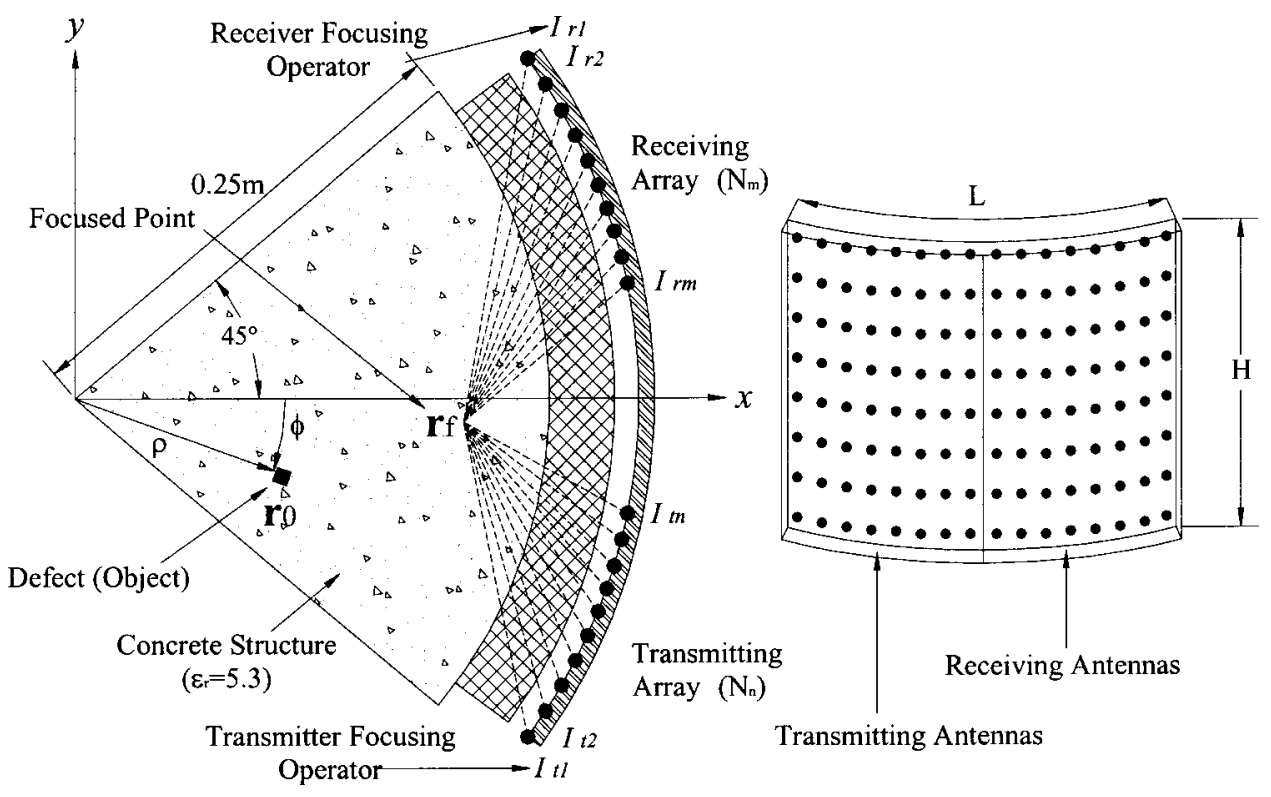

Fig. 1. Use of microwave arrays to focus waves on subsurface point

arrays). This study proposes a unique bifocusing technique that focuses not only the conventional receiving arrays but also the transmitting arrays in order to improve the image resolution. The bifocusing technique enables the microwave to be concentrated on a small region inside the reconstructing area, making it possible to investigate the area precisely. All the elements of the arrays are weighted by a focusing operator so as to be focused on

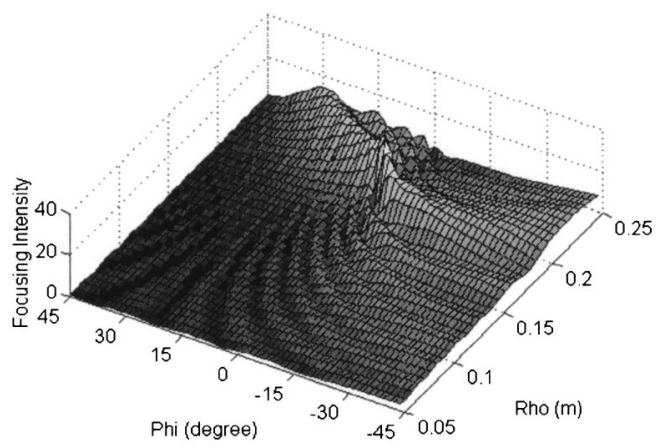

(a) Transmitting Intensity

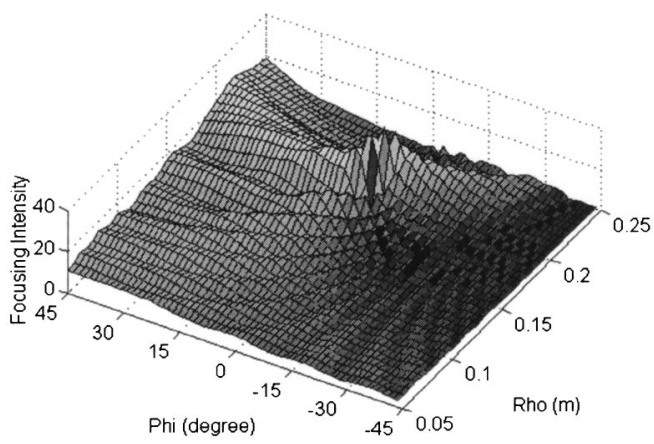

(b) Receiving Intensity

Fig. 2. Focusing intensity of transmitting and receiving signals (focused point: $\rho=0.20 \mathrm{~m}, \phi=0^{\circ}$ ) a unique object point. This can be achieved by a numerical treatment of the measurement matrix.

The focusing operator can be obtained by taking an inverse of the electric field induced by a current line. It is well known that the electric fields of the infinite electric line source are proportional to a Hankel function of the second kind whose argument is proportional to the distance from the source to the observation point (Balanis 1989). Therefore, the incident field $E_{i}$ at $\mathbf{r}_{i}$ $=\left(x_{i}, y_{i}\right)$ when focusing on every transmitting point $\mathbf{r}_{t n}$ $=\left(x_{t n}, y_{t n}\right)$ on the reconstructing point $\mathbf{r}_{f}=\left(x_{f}, y_{f}\right)$ can be expressed as

$$
E_{i}\left(\mathbf{r}_{i}\right)=\sum_{n=1}^{N_{n}} I_{T n}\left(\mathbf{r}_{f}\right) H_{0}^{(2)}\left(k_{e}\left|\mathbf{r}_{t n}-\mathbf{r}_{i}\right|\right)
$$

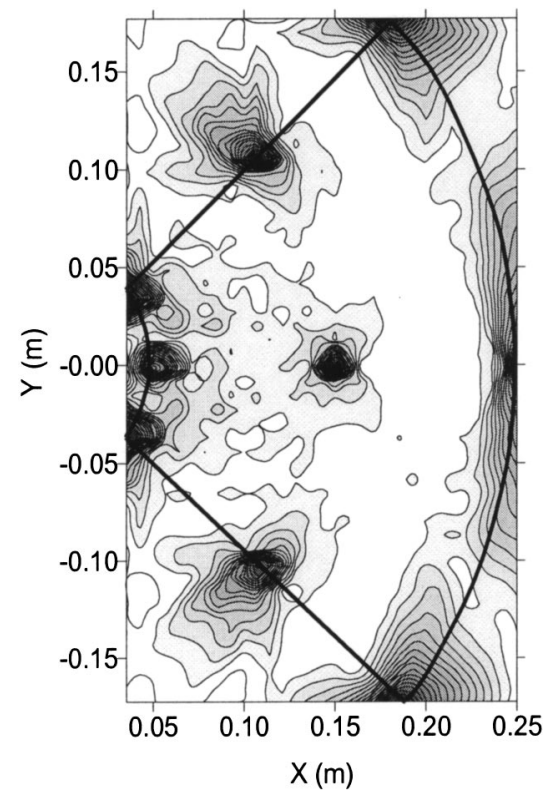

Fig. 3. Image of nine pointlike objects 


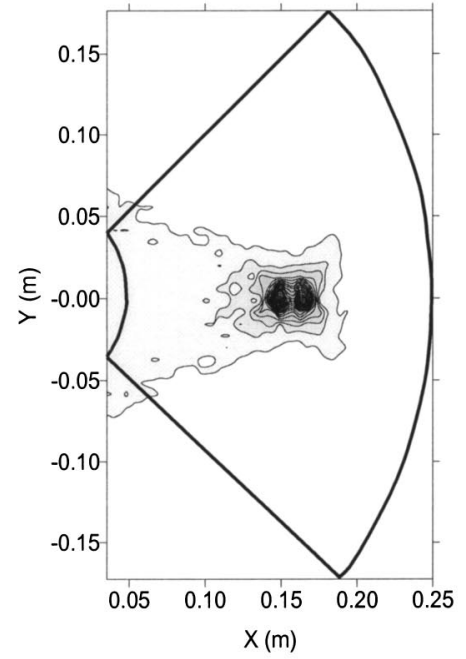

(a) Transverse Distance of $0.013 \mathrm{~m}$ (Bi-Focusing)

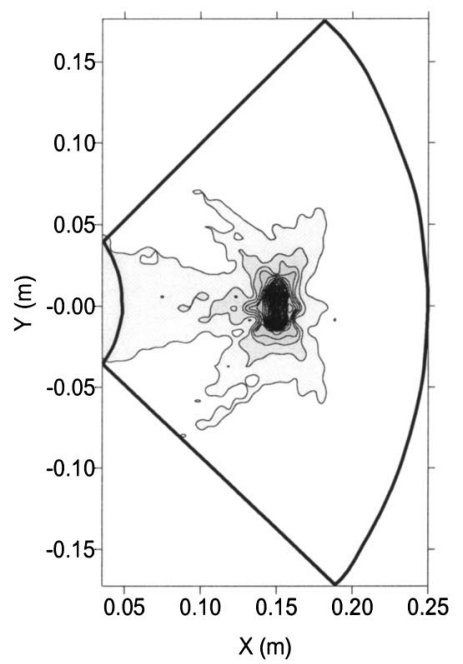

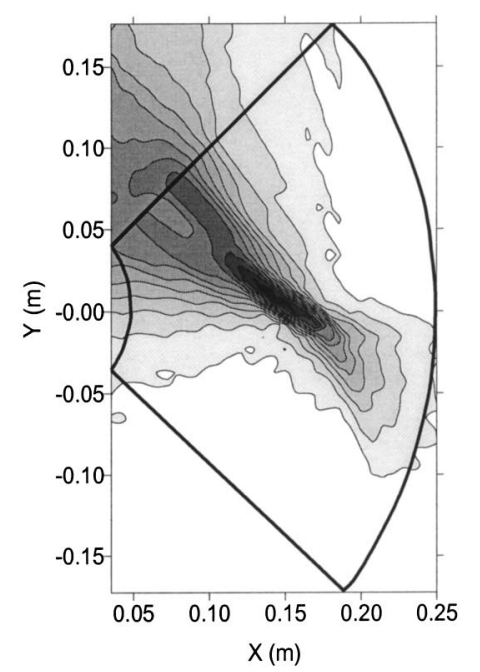

(b) Transverse Distance of $0.013 \mathrm{~m}$ (Mono-Focusing)

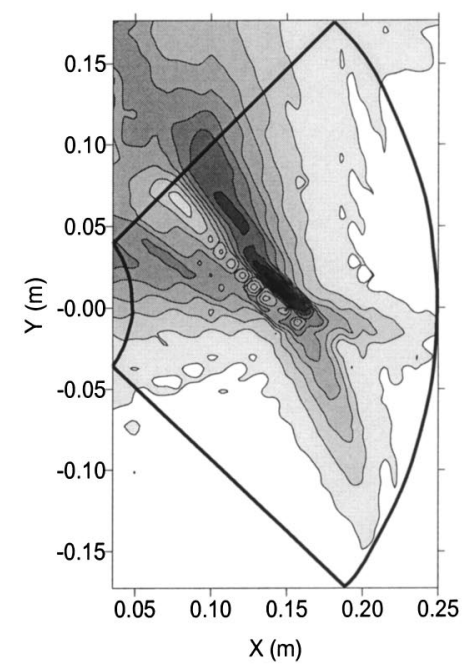

(c) Longitudinal Distance of $0.013 \mathrm{~m}$ (Bi-Focusing) (d) Longitudinal Distance of $0.013 \mathrm{~m}$ (Mono-Focusing)

Fig. 4. Reconstructed image of two pointlike objects

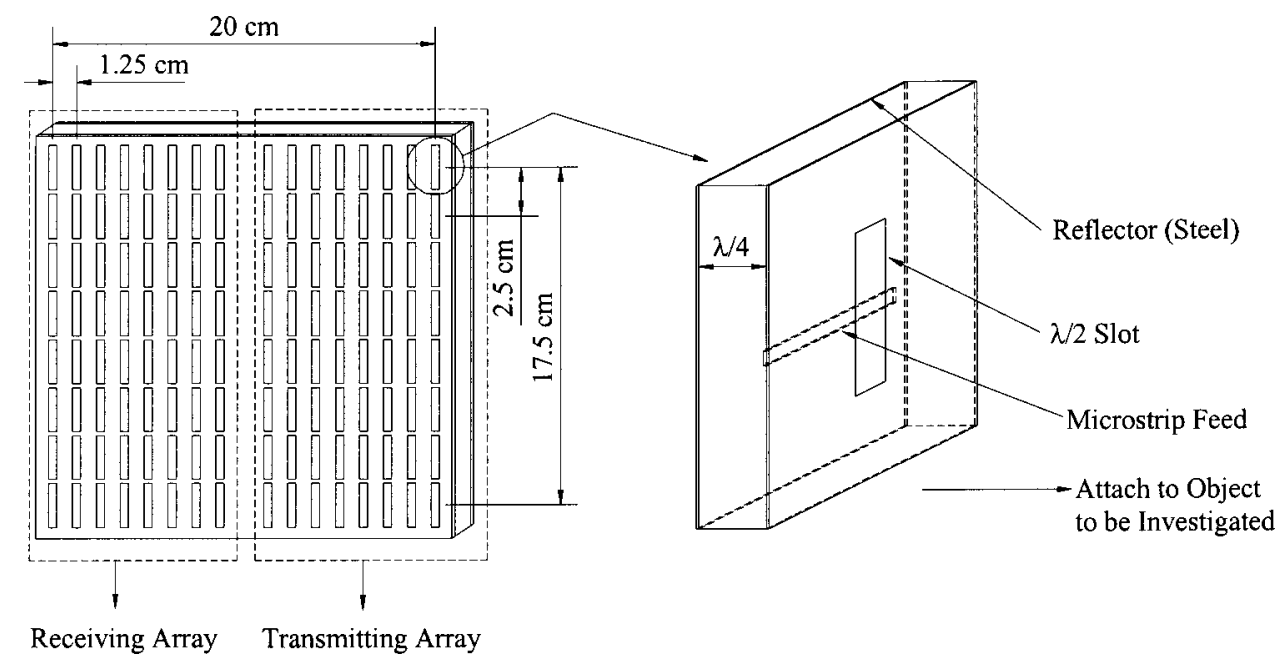

Fig. 5. Conceptual design of planar slot antenna array 


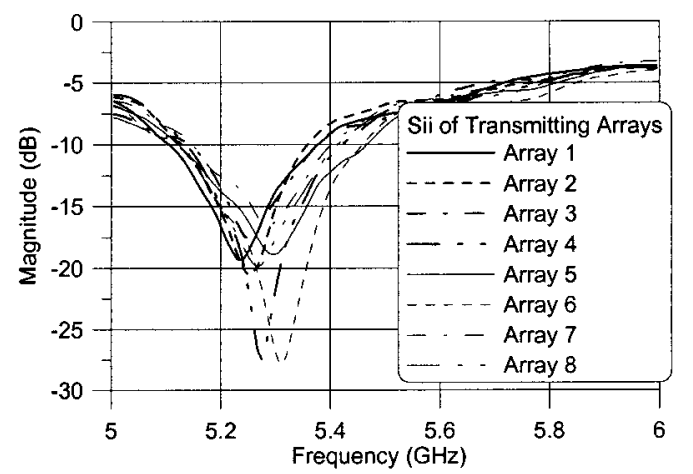

(a) $\mathrm{S}_{\mathrm{ij}}$ Measurement of Transmitting Arrays

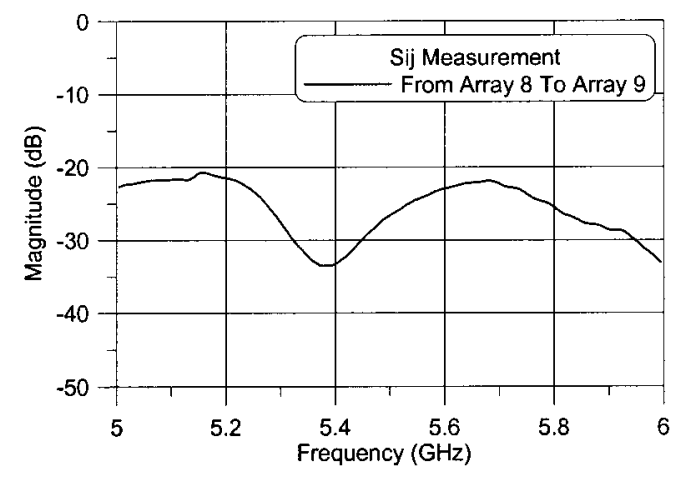

(b) Transmission Measurement ( $\mathrm{S}_{\mathrm{ij}}$ of Array 8 and Array 9$)$

Fig. 6. Measurement results of antenna array

where $I_{T n}\left(\mathbf{r}_{f}\right)$, focusing operator, is given by

$$
I_{T n}\left(\mathbf{r}_{f}\right)=\frac{1}{H_{0}^{(2)}\left(k_{e}\left|\mathbf{r}_{t n}-\mathbf{r}_{f}\right|\right)}
$$

where $k_{e}=k_{0} \sqrt{\epsilon_{r}}$. The concrete was assumed to be homogeneous having uniform dielectric constant $\epsilon_{r}=5.3$ for the purpose of computational ease.

Scattered field measured at $\mathbf{r}_{r m}=\left(x_{r m}, y_{r m}\right)$ of a defect (object) placed at $\mathbf{r}_{0}=\left(x_{0}, y_{0}\right)$ is

$$
E_{s}\left(\mathbf{r}_{r m}\right)=E_{i}\left(\mathbf{r}_{0}\right) I_{\mathrm{obj}} \times H_{0}^{(2)}\left(k_{e}\left|\mathbf{r}_{r m}-\mathbf{r}_{0}\right|\right)
$$

where $I_{\mathrm{obj}}=$ constant for the object containing its electromagnetic macroscopic characteristics.

When focusing back the received field at $\mathbf{r}_{r m}=\left(x_{r m}, y_{r m}\right)$ on the interest point $\mathbf{r}_{f}=\left(x_{f}, y_{f}\right)$, electromagnetic image of $E_{f}\left(\mathbf{r}_{f}\right)$ at $\mathbf{r}_{f}=\left(x_{f}, y_{f}\right)$ can be expressed as

$$
E_{f}\left(\mathbf{r}_{f}\right)=\sum_{m=1}^{N_{m}} E_{s}\left(\mathbf{r}_{r m}\right) I_{R m}\left(\mathbf{r}_{f}\right)
$$

where $I_{R m}\left(\mathbf{r}_{f}\right)$, focusing operator, is given by

$$
I_{R m}\left(\mathbf{r}_{f}\right)=\frac{1}{H_{0}^{(2)}\left(k_{e}\left|\mathbf{r}_{r m}-\mathbf{r}_{f}\right|\right)}
$$

Finally, all the process can be grouped as follows:

$$
\begin{aligned}
E_{f}\left(\mathbf{r}_{f}\right)= & \sum_{m=1}^{N_{m}}\left[\frac{1}{H_{0}^{(2)}\left(k_{e}\left|\mathbf{r}_{r m}-\mathbf{r}_{f}\right|\right)}\right. \\
& \times \sum_{i=1}^{N_{i}}\left\{I_{\mathrm{obj}, i} \times H_{0}^{(2)}\left(k_{e}\left|\mathbf{r}_{r m}-\mathbf{r}_{0, i}\right|\right)\right. \\
& \left.\left.\times \sum_{n=1}^{N_{n}} \frac{H_{0}^{(2)}\left(k_{e}\left|\mathbf{r}_{t n}-\mathbf{r}_{0, i}\right|\right)}{H_{0}^{(2)}\left(k_{e}\left|\mathbf{r}_{t n}-\mathbf{r}_{f}\right|\right)}\right\}\right]
\end{aligned}
$$

or

$$
\begin{aligned}
E_{f}\left(\mathbf{r}_{f}\right)= & {\left[\begin{array}{llll}
I_{T 1} & I_{T 2} & \cdots & I_{T n}
\end{array}\right] \cdot\left[\begin{array}{cccc}
E_{s, T 1 R 1} & E_{s, T 1 R 2} & \cdots & E_{s, T 1 R m} \\
E_{s, T 2 R 1} & E_{s, T 2 R 2} & \cdots & E_{s, T 2 R m} \\
\vdots & \vdots & \ddots & \vdots \\
E_{s, T n R 1} & E_{s, T n R 2} & \cdots & E_{s, T n R m}
\end{array}\right] } \\
& \times\left[\begin{array}{c}
I_{R 1} \\
I_{R 2} \\
\vdots \\
I_{R m}
\end{array}\right]
\end{aligned}
$$

\section{Reconstruction Parameters}

The formulation derived in the previous section was applied to a cylindrical antenna array consisting of 32 transmitting and 32 receiving antennas, with the illuminating microwave frequency of 10.0 GHz. At this frequency, the effective wavelength in concrete, $\lambda_{e}$, is $1.3 \mathrm{~cm}$, which is calculated from $\lambda_{e}=\lambda_{0} / \sqrt{\epsilon_{r}}$. Figs. 2(a and $b$ ), respectively show the focusing intensity of transmitting and receiving signals when focused on the point of $(\rho=0.20 \mathrm{~m}$, $\left.\phi=0^{\circ}\right)$, which is $(x=0.20 \mathrm{~m}, y=0 \mathrm{~m})$ in rectangular coordinates. The focusing intensity reaches the maximum value (32) at the focused point. This value is consistent with the number of transmitting and receiving antennas.

In order to study the focusing capability of the system at different distances and view angles, nine point-like objects were

Table 1. Descriptions of Cases for Numerical and Experimental Study

\begin{tabular}{llcc}
\hline $\begin{array}{l}\text { Case } \\
\text { number }\end{array}$ & \multicolumn{1}{c}{ Description } & $\begin{array}{c}\text { Reconstructed area }(x \times y) \\
(\mathrm{cm} \times \mathrm{cm})\end{array}$ & $\begin{array}{c}\text { Exact locations ofobjects }(x, y) \\
(\mathrm{cm})\end{array}$ \\
\hline 1 & $\begin{array}{l}\text { Steel sphere at the center of concrete panel } \\
2\end{array}$ & $4 \times 20$ & $(0,0)$ \\
3 & Square air void inside concrete & $8 \times 20$ & $(4,0)$ \\
$(4,0)$ & $8 \times 20$ & $(5,1.25)(5,-1.25)$ \\
$4^{\mathrm{a}}$ & Rectangular air void inside concrete & & $10 \times 20$ \\
& two steel bars & & $(5,0)(7.5,0)$ \\
$5^{\mathrm{a}}$ & With transverse distance of $2.5 \mathrm{~cm}\left(\lambda_{e}\right)$ & $10 \times 20$ & \\
\hline
\end{tabular}

${ }^{\mathrm{a} O n l y}$ used for numerical simulation. 


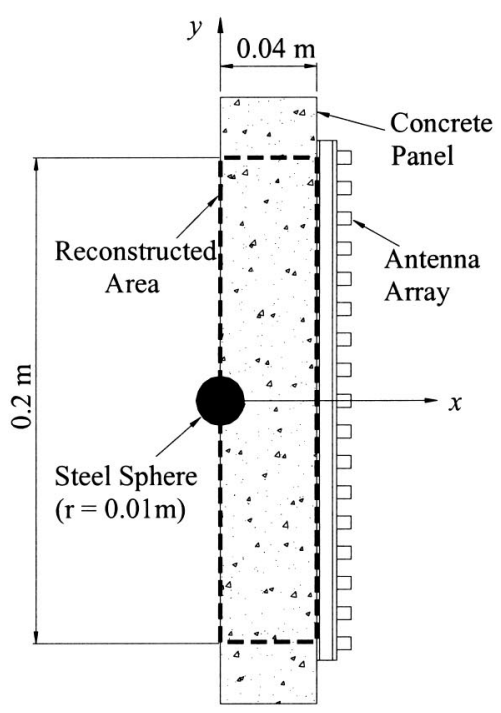

(a) Description of Reconstructed Area

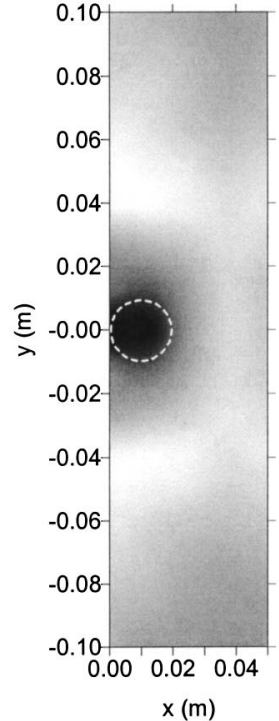

(b) Simulation Result

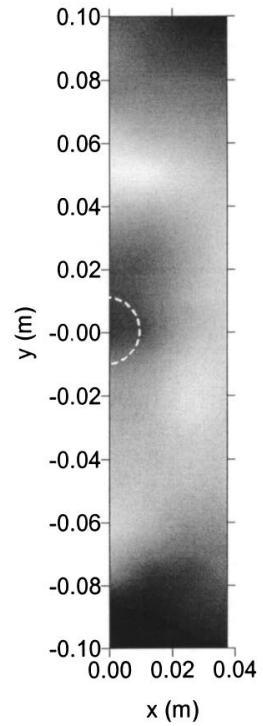

(c) Experimental Result

Fig. 7. Description and reconstructed image of Case 1

placed at the boundary and the central landmark points of the reconstructing $2 \mathrm{D}$ cross section. The results in Fig. 3 show a good uniformity in the focusing intensity levels at the nine points. The impulsive shapes of the focusing intensity at the focused point suggest a desirable behavior of the reconstruction algorithm.

In addition, simulations were conducted using numerical measurements to verify the resolution capability of the system. Two pointlike objects were placed with the transverse and the longitudinal distances of $1.3 \mathrm{~cm}$ at the center of the reconstructing 2D cross section. Fig. 4 shows the comparison of the use of bifocusing and monofocusing techniques. The results demonstrate that the system, due to the use of bifocusing (focusing both in the transmitting and receiving arrays), is able to achieve a resolution, both in the transversal and in the longitudinal directions, in the order of the effective wavelength in the dielectric medium, which is $1.3 \mathrm{~cm}$ at $10.0 \mathrm{GHz}$.

\section{Design and Test of Slot Antenna Array}

A prototype planer antenna array was developed for proof-ofconcept study. An illuminating frequency of $5.2 \mathrm{GHz}$ was chosen (beyond which the fabrication of the antenna array by hands in a university laboratory would become difficult). The image resolution is $2.5 \mathrm{~cm}$ at this frequency in concrete. Among a few alternatives, a micro-strip slot antenna was chosen due to its ability to

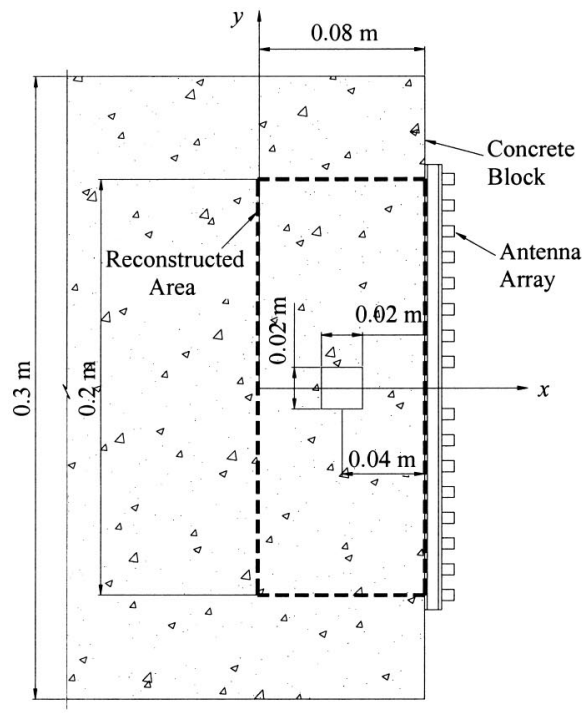

(a) Description of Reconstructed Area

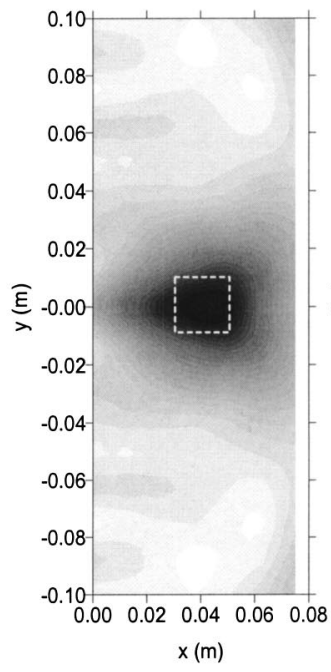

(b) Simulation Result

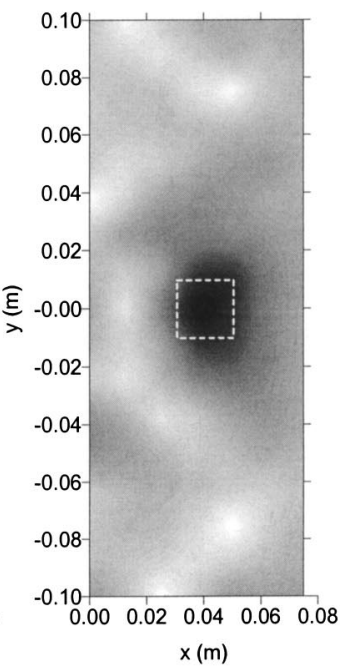

(c) Experimental Result

Fig. 8. Description and reconstructed image of Case 2 


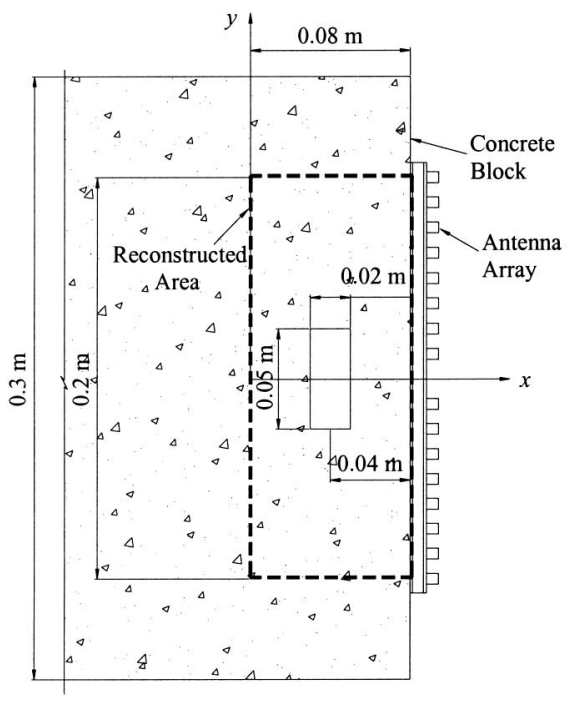

(a) Description of Reconstructed Area

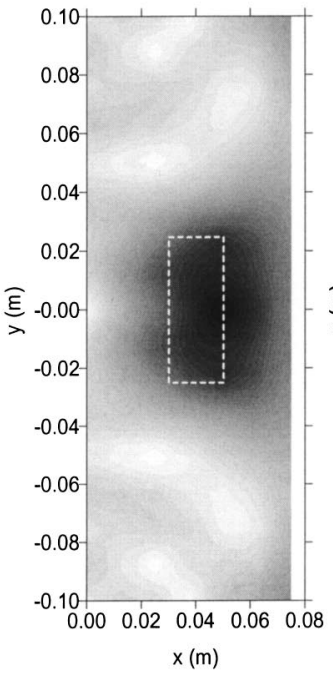

(b) Simulation Result

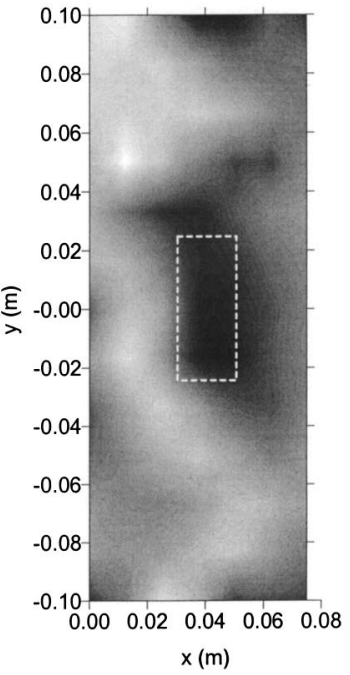

(c) Experimental Result

Fig. 9. Description and reconstructed image of Case 3

produce bidirectional or unidirectional radiation patterns with a large bandwidth and its additional degree of freedom in the antenna design (Garg et al. 2001). Fig. 5 illustrates the antenna geometry designed in this study. Each of the transmitting and receiving antenna arrays consists of $64(8 \times 8)$ antenna slots and the total dimension of the two arrays is $20 \mathrm{~cm} \times 20 \mathrm{~cm}$. This design represents a reasonable tradeoff between the resolution and the reconstruction area covered by the antenna array (Kim 2002).

A significant technical challenge in developing the antenna array is to achieve a high radiation performance (meaning a larger bandwidth at the illuminating frequency) and low mutual coupling (meaning low interference among the slot antenna elements). Although numerous studies have been performed by elec- trical engineers to study radiation of antenna arrays into air for communication purposes, no literature can be found regarding design knowledge for concrete-radiation antenna arrays. The antenna array fabricated in this study was tested using a network analyzer, measuring reflection parameters $\left(S_{i i}\right)$ for investigating the radiation performance and transmission parameters $\left(S_{i j}\right)$ for the mutual coupling. The antenna array was placed on a concrete block, allowing the wave radiating through the concrete. As plotted in Fig. 6(a), the magnitude of $S_{i i}$ parameters around $5.2 \mathrm{GHz}$ is less than $-10 \mathrm{~dB}$, implying that the antenna achieved high radiation performance. The transmission parameters plotted in Fig. 6(b) shows that the interference between the colateral elements (Arrays 8 and 9) is as low as $-20 \mathrm{~dB}$, which is acceptable.

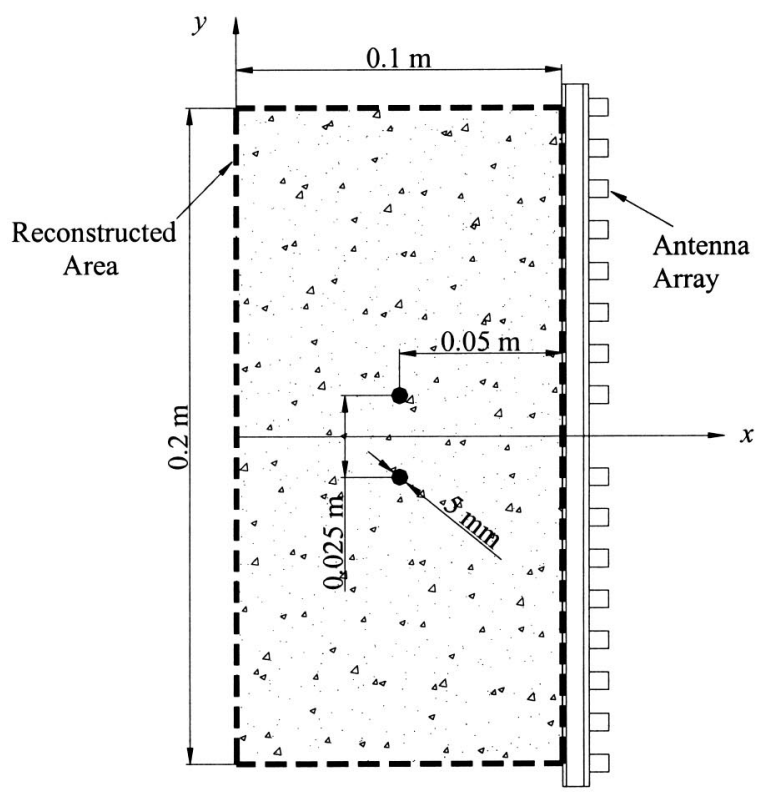

(a) Description of Reconstructed Area

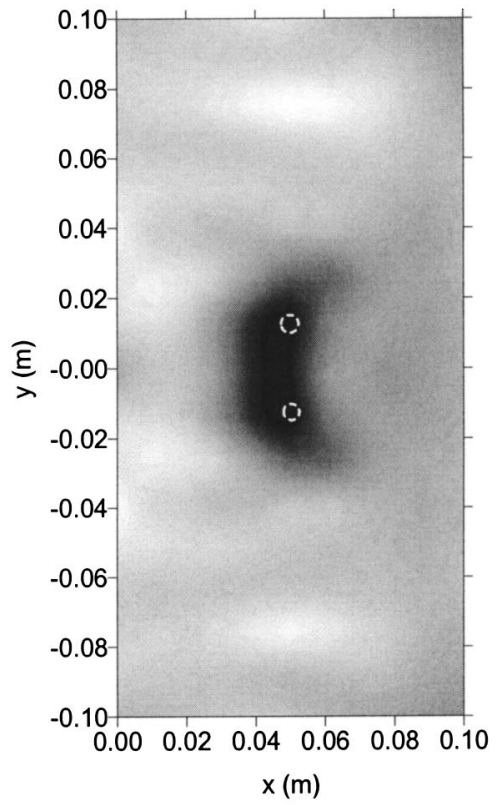

(b) Simulation Result

Fig. 10. Description and reconstructed image of Case 4 


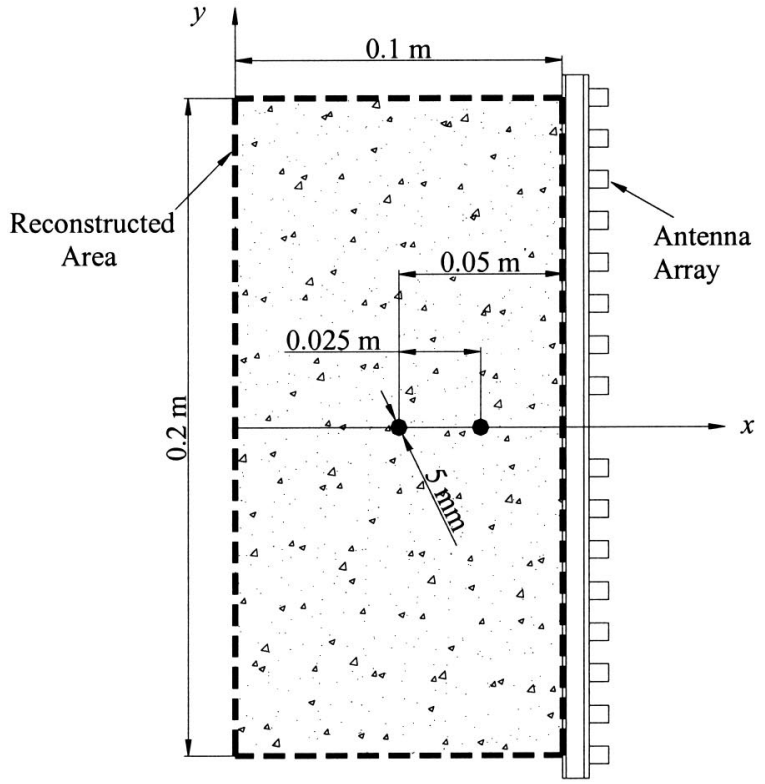

(a) Description of Reconstructed Area

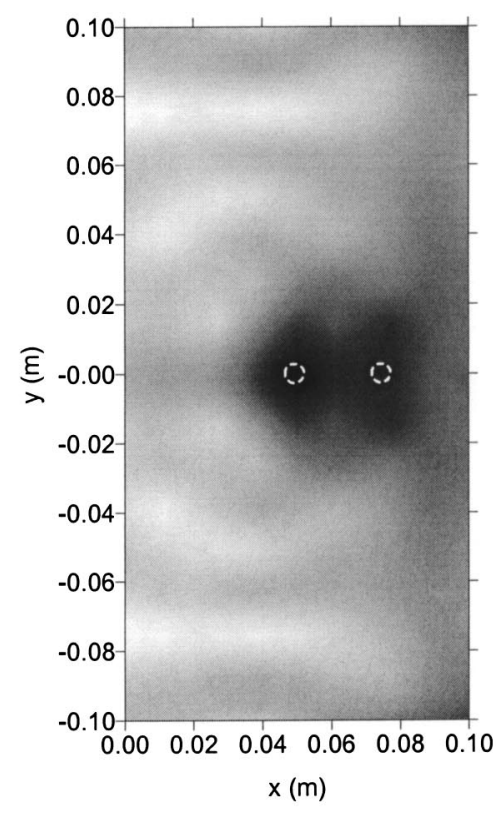

(b) Simulation Result

Fig. 11. Description and reconstructed image of Case 5

Therefore, this study achieved a high-performance antenna array.

\section{Numerical Simulation}

Numerical simulation was carried out to predict the effectiveness of the proposed microwave imaging technology in imaging a concrete specimen with internal air voids and steel bars. Totally five cases, as listed in Table 1, were modeled with $5.2 \mathrm{GHz}$ as the illuminating frequency. Some were compared with the results using $10.0 \mathrm{GHz}$ as the illuminating frequency. For the simulation, a planar rectangular antenna array was used with $8 \times 8$ transmitting and $8 \times 8$ receiving antennas for the case of $5.2 \mathrm{GHz}$ (same as the one fabricated in this study) and $16 \times 16$ transmitting and $16 \times 16$ receiving antennas for the case of $10.0 \mathrm{GHz}$ (due to the fact that a higher frequency requires a more densely placed antenna array). For the direct problem in which the antenna transmits wave and receives scattered field, a 3D structure simulator, CST Microwave Studio (CST GmbH, Germany) (CST-Computer Simulation Technology 2002), was used for measuring the transmission parameters $\left(S_{i j}\right)$ in each array. CST Microwave Studio is a tool for the 3D electromagnetic simulation of high frequency problems. In the simulation, the background material was modeled as concrete and open boundary conditions were applied at the top and the bottom of the geometry in order to generate absorbing conditions. The reconstructed images for each case are reported in Figs. 7-11 along with the description of the reconstructed area.

In Case 1, the steel sphere of $0.04 \mathrm{~m}$ diameters was successfully reconstructed with the exact location and dimension as shown in Fig. 7(b). The reconstructed area was limited to $5 \mathrm{~cm}$ in $x$-direction because the open boundary in CST Microwave Studio (CST GmbH, Germany) was set at the $y$-axis, from which the distance to the antenna array is $5 \mathrm{~cm}$. The reconstructed areas in the $x$-direction in the other cases were also determined by the location of the open boundary. In Cases 2 and 3, a square and a rectangular air voids were placed inside concrete material, respec- tively. The reconstructed images as shown in Figs. 8(b) and 9(b) show the exact locations and the approximate sizes of the air voids in both cases, although there were some noises. Cases 4 and 5 were designed to verify the image resolution. As shown in Fig. 10(b), the two steel bars placed $2.5 \mathrm{~cm}\left(\lambda_{e}\right)$ apart in the transverse direction were clearly detected, although the resolution was not sufficient enough to reconstruct the exact shapes of the bars. Fig. 11(b) shows similar resolution in the longitudinal direction. From the results of Cases 4 and 5, it was demonstrated that the image resolution is in the order of the wavelength in concrete $(2.5$ $\mathrm{cm}$ ), which can identify approximately the shapes and locations of the voids.

A better resolution, however, can be achieved by increasing the illuminating frequency. In order to demonstrate this, Cases 2 and 3 were investigated again with $10.0 \mathrm{GHz}$ as an illuminating frequency and the results are compared in Fig. 12. Using 10.0 $\mathrm{GHz}$, the image resolution was clearly improved, indicating exactly the shapes as well as the locations of the voids.

\section{Experimental Verification}

\section{Experimental Setup}

The effectiveness of the proposed subsurface imaging technology was further investigated through a series of experiments on a concrete panel and a concrete block. A steel rod was placed on one of the faces of the concrete panel to simulate steel rebar. The $30 \mathrm{~cm} \times 30 \mathrm{~cm} \times 30 \mathrm{~cm}$ concrete block involves two internal voids artificially generated using Styrofoam (whose dielectric property is the same as that of the air); one is a $2 \mathrm{~cm}$ cubic void and the other is a $5 \mathrm{~cm} \times 2 \mathrm{~cm} \times 2 \mathrm{~cm}$ void. The Styrofoam blocks were inserted into the concrete during the pouring of concrete with the distance of $3 \mathrm{~cm}$ from the face of concrete to the face of Styrofoam in both cases. Samples using steel bars in the air were also prepared. All the experimental cases, identical to those used in the numerical simulation, were described in Table 1. 


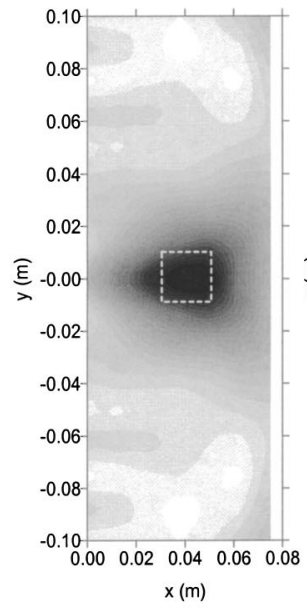

(a) Result of Case 2 Using $5.2 \mathrm{GHz}$ (left) and $10.0 \mathrm{GHz}$ (right)
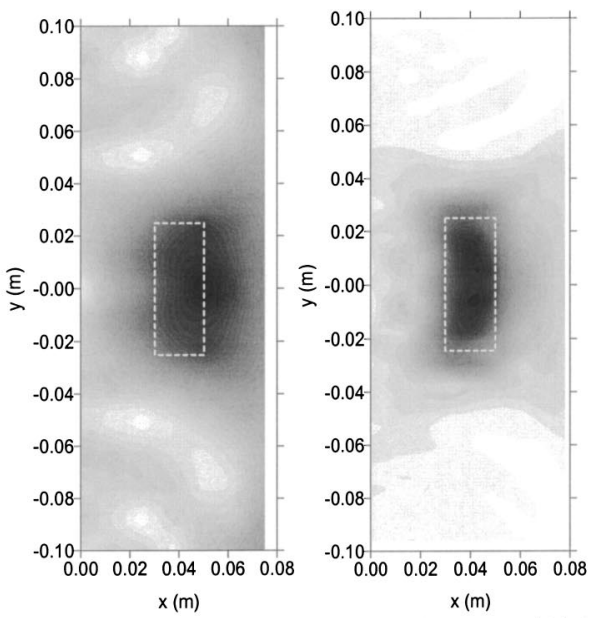

(b) Result of Case 3 Using $5.2 \mathrm{GHz}$ (left) and $10.0 \mathrm{GHz}$ (right)

Fig. 12. Resolution improvement using higher frequency

The experimental setup consisted of a network analyzer, a switch box, coaxial cables, and the antenna array as shown in Fig. 13. The network analyzer is a two-port device capable of transmitting a signal at one port and receiving a signal at the other port while evaluating the magnitude and phase of the receiving signal. The network analyzer was used to evaluate the signal transmitted through the medium $\left(S_{12}\right)$. The switch box developed in this study is a radio frequency network capable of controlling multiple antennas in the array and selecting them individually to perform $S_{12}$ measurement. Calibration was performed to remove the effect of wave reflections and loss in the coaxial cables and the switch box from the measured signals (Kim 2002; Kim et al. 2002).

\section{Experimental Results}

A continuous 5.2 GHz sinusoidal electromagnetic (EM) wave was generated from the signal analyzer and sent to the test specimen. Transmission measurements of each transmitting and receiving array pair at this frequency were assembled to form a measurement matrix. The switch box controlled the active location of the transmitting and receiving arrays from $S_{1,9}$ to $S_{8,16}$. Each measured signal was divided by the calibration factor at $5.2 \mathrm{GHz}$ and assembled to form a calibrated measurement matrix to be multiplied by the numerical focusing operators. The reconstructed images, the distributions of the electric current amplitude, are

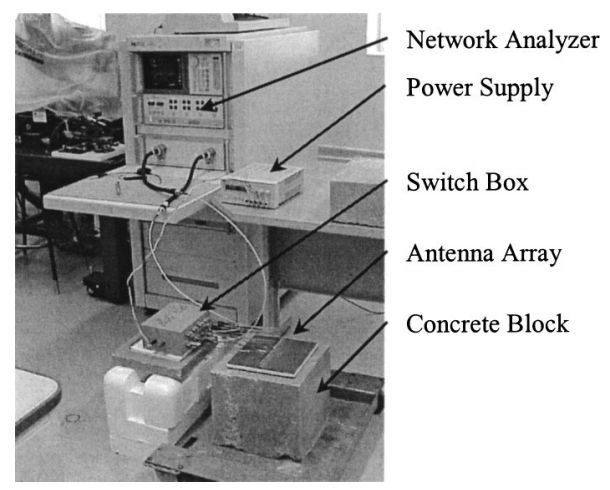

Fig. 13. Experimental setup plotted in Figs. 7-9 for the center cuts of all the experimental cases.

In Case 1 that is identical to numerical simulation Case 1, the location as well as the size of the steel sphere was detected, although the edge effects due to the small size of concrete specimen added some noises to the experimental result. Experimental results of Cases 2 and 3 are shown in Figs. 8(a) and 9(a), that involve air voids inside the concrete block, respectively, identical to simulation Cases 2 and 3. The images of the square and the rectangular Styrofoam's (i.e., the air voids) were successfully reconstructed in terms of their approximate sizes and locations, although the resolution $(2.5 \mathrm{~cm}$ at $5.2 \mathrm{GHz})$ was not sufficient to reconstruct the exact features. The numerical simulation results in Figs. 8(b) and 9(b) agree with the experimental results in Figs. 8(c) and 9(c). This verifies the effectiveness of the simulation model using the program, CST Microwave Studio.

\section{Conclusions}

A microwave subsurface imaging technology using antenna arrays and software focusing was developed in this study for detecting invisible damage and/or objects inside concrete structures. The following conclusions can be drawn from the simulation and experimental analysis:

1. The imaging reconstruction algorithm using the bifocusing numerical operator developed in this study resulted in uniformed focusing intensity. A resolution in the order of the wavelength in the dielectric medium (concrete in this study) was achieved by focusing both the transmitting and receiving arrays;

2. The prototype slot antenna array consisting of $8 \times 8$ transmitting and $8 \times 8$ receiving antennas designed and fabricated in this study achieved the goal of high radiation and low mutual coupling;

3. The microwave imaging system consisting of the antenna array integrated with the imaging reconstruction algorithm is capable of detecting air voids and steel inside concrete; and

4. The effectiveness of simulation using CST Microwave Studio was experimentally verified. The simulation demonstrated that the image resolution can be improved by increasing the illuminating frequency. 


\section{Acknowledgment}

This study was supported by California Department of Transportation under Award No. 65A0140.

\section{Notation}

The following symbols are used in this paper:

$E_{i}=$ incident electric field;

$E_{s}=$ scattered electric field;

$J=$ electric current distribution;

$J_{\text {eq }}=$ equivalent electric current distribution;

$k_{e}=$ effective wave number in medium;

$k_{0}=$ wave number in air;

$S_{i i}=$ scattering parameter (reflection parameter);

$S_{i j}=$ scattering parameter (transmission parameter);

$\boldsymbol{\epsilon}_{r}=$ relative dielectric constant;

$\lambda_{e}=$ effective wave length in medium; and

$\lambda_{0}=$ wave length in air.

\section{References}

Balanis, C. A. (1989). Advanced engineering electromagnetics, Wiley, New York.

Bois, K., Benally, A., and Zoughi, R. (1998). "Near-field microwave reflection property analysis of concrete using open-ended rectangular waveguides at S- and X-band." Proc. SPIE, 3396, 37-46.

Computer Simulation Technology (CST). (2002). HF design and analysis-CST microwave studio 4, Software Manual, CST GmbH, Germany.

Feng, M. Q., Flaviis, F. D., and Kim, Y. J. (2002). "Use of microwaves for damage detection of fiber reinforced polymer-wrapped concrete structures." J. Eng. Mech., 128(2), 172-183.

Garg, R., Bhartia, P., Bahl, I., and Ittipiboon, A. (2001). Microstrip antenna design handbook, Artech House, Boston.

Gironés, I. M., Jofre, L., Ferrando, M., De Los Reyes, M., and Bolomey, J. Ch. (1987). "Microwave imaging with crossed linear arrays." IEE Proc.: Optoelectron., 134(3), 249-252.

Huston, D., Hu, J. Q., Maser, K., Weedon, W., and Adam, C. (2000). "GIMA ground penetrating radar system for monitoring concrete bridge decks." J. Appl. Geophys., 43, 139-146.

Kharkovsky, S. N., and Atis, C. D. (2003). "Nondestructive testing of mortar specimens using the microwave free-space method." J. Mater. Civ. Eng., 15(2), 200-204.

Kim, Y. J. (2002). Development of electromagnetic imaging technology for damage detection, $\mathrm{PhD}$ dissertation, Univ. of California, Irvine, Irvine, Calif.

Kim, Y. J., Jofre, L., De Flaviis, F., and Feng, M. Q. (2002). "Microwave sub-surface imaging technology for damage detection of concrete structures." Proc. of 15th ASCE Engineering Mechanics Division Conf., New York, Columbia University, New York, 589-596.

Rhim, H. C., and Büyüköztürk, O. (2000). "Wideband microwave imaging of concrete for nondestructive testing." J. Struct. Eng., 126(12), $1451-1457$. 
Copyright of Journal of Engineering Mechanics is the property of American Society of Civil Engineers and its content may not be copied or emailed to multiple sites or posted to a listserv without the copyright holder's express written permission. However, users may print, download, or email articles for individual use. 